\title{
Recent results from PHOBOS on particle production at high $p_{T}$
}

\section{PHOBOS Collaboration}

B. Alver ${ }^{4}$, B.B. Back ${ }^{1}$, M.D. Baker ${ }^{2}$, M. Ballintijn ${ }^{4}$, D.S. Barton ${ }^{2}$, R.R. Betts ${ }^{6}$, A.A. Bickley ${ }^{7}$, R. Bindel ${ }^{7}$, W. Busza ${ }^{4}$, A. Carroll ${ }^{2}$, Z. Chai ${ }^{2}$, V. Chetluru ${ }^{6}$, M.P. Decowski ${ }^{4}$, E. García ${ }^{6}$, T. Gburek ${ }^{3}$, N. George ${ }^{2}$, K. Gulbrandsen $^{4}$, C. Halliwell ${ }^{6}$, J. Hamblen ${ }^{8}$, I. Harnarine ${ }^{6}$, M. Hauer ${ }^{2}$, C. Henderson ${ }^{4}$, D.J. Hofman ${ }^{6}$, R.S. Hollis ${ }^{6}$, R. Holyński ${ }^{3}$, B. Holzman ${ }^{2}$, A. Iordanova ${ }^{6}$, E. Johnson ${ }^{8}$, J.L. Kane ${ }^{4}$, N. Khan ${ }^{8}$, P. Kulinich ${ }^{4}$, C.M. Kuo ${ }^{5}$, W. Li ${ }^{4}$, W.T. Lin ${ }^{5}$, C. Loizides $^{4}$, S. Manly ${ }^{8}$, A.C. Mignerey ${ }^{7}$, R. Nouicer ${ }^{2}$, A. Olszewski ${ }^{3}$, R. Pak ${ }^{2},{\text { C. } \text { Reed }^{4} \text {, E. Richardson }}^{7}$, C. Roland $^{4}$, G. Roland ${ }^{4}$, J. Sagerer ${ }^{6}$, H. Seals ${ }^{2}$, I. Sedykh ${ }^{2}$, C.E. Smith ${ }^{6}$, M.A. Stankiewicz ${ }^{2}$, P. Steinberg ${ }^{2}$, G.S.F. Stephans ${ }^{4}$, A. Sukhanov ${ }^{2}$, A. Szostak ${ }^{2}$, M.B. Tonjes ${ }^{7}$, A. Trzupek ${ }^{3}$, C. Vale $^{4}$, G.J. van Nieuwenhuizen ${ }^{4}$, S.S. Vaurynovich ${ }^{4}$, R. Verdier $^{4}$, G.I. Veres ${ }^{4,9, a, b}$, P. Walters ${ }^{8}$, E. Wenger ${ }^{4}$, D. Willhelm ${ }^{7}$, F.L.H. Wolfs ${ }^{8}$, B. Wosiek ${ }^{3}$, K. Woźniak ${ }^{3}$, S. Wyngaardt ${ }^{2}$, B. Wysłouch ${ }^{4}$

${ }^{1}$ Argonne National Laboratory, Argonne, IL 60439-4843, USA

${ }^{2}$ Brookhaven National Laboratory, Upton, NY 11973-5000, USA

${ }^{3}$ Institute of Nuclear Physics PAN, Kraków, Poland

${ }^{4}$ Massachusetts Institute of Technology, Cambridge, MA 02139-4307, USA

${ }^{5}$ National Central University, Chung-Li, Taiwan

${ }^{6}$ University of Illinois at Chicago, Chicago, IL 60607-7059, USA

${ }^{7}$ University of Maryland, College Park, MD 20742, USA

${ }^{8}$ University of Rochester, Rochester, NY 14627, USA

${ }^{9}$ Present address: Department of Atomic Physics, Eötvös Loránd University, Budapest 1117, Hungary

Received: 15 September 2008 / Revised: 6 January 2009 / Published online: 10 February 2009

(C) Springer-Verlag / Società Italiana di Fisica 2009

\begin{abstract}
A selection of experimental results from the PHOBOS Collaboration relevant for probing high-energy nuclear collisions with high transverse momentum particles is presented. The inclusive yields of charged particles and comparisons between nuclear and elementary collisions already reveal a large amount of parton energy loss in the hot and dense medium created in heavy ion collisions. Remarkable scaling and factorization features are observed, unifying the data taken at various collision energies, centralities and nuclear sizes. To further analyze the nature of the energy loss, a measurement of pseudorapidity $(\Delta \eta)$ and azimuthal angle $(\Delta \phi)$ correlations between high transverse momentum charged hadrons $\left(p_{T}>2.5 \mathrm{GeV} / c\right)$ and all associated charged particles is presented at both shortrange (small $\Delta \eta$ ) and long-range (large $\Delta \eta$ ) over a continuous detector acceptance covering $-4<\Delta \eta<2$. Various near- and away-side features of the correlation structure are discussed as a function of centrality in $\mathrm{Au}+\mathrm{Au}$ collisions at $\sqrt{s_{N N}}=200 \mathrm{GeV}$. The results provide new information about the longitudinal $(\Delta \eta)$ extent of the near-side 'ridge' structure, first observed by the STAR Collaboration over a narrower $\eta$ range. In central $\mathrm{Au}+\mathrm{Au}$ collisions the ridge structure extends to at least $\Delta \eta=4$, and its strength
\end{abstract}

completely diminishes as collisions become more peripheral.

\section{Introduction}

The general goal of the experimental studies of high energy nuclear collisions is to create a new phase of matter at very high density and temperature, where hadrons are replaced by partons as relevant degrees of freedom. The conditions in the created medium are thought to be similar to the situation in the early Universe well before the nucleosynthesis. Large part of the experimental evidence that a new form of matter is created in these collisions comes from 'hard probes', especially from measurements related to particles with high transverse momentum. The interaction between those particles and the produced medium is considered to be a useful tool to reveal certain features of the elusive new form of matter.

\footnotetext{
a e-mail: gabor.veres@cern.ch

${ }^{b}$ Presenter of the paper on the 3rd International Conference on Hard and Electromagnetic Probes of High-Energy Nuclear Collisions, 8-14 June 2008, Illa da Toxa (Galicia-Spain).
} 
After a brief description of the PHOBOS detector system, two particular methods to study these interactions will be presented. The first one is the comparison of inclusive charged hadron transverse momentum spectra between elementary and nuclear collisions, between various collision energies and centralities. The second approach utilizes the large acceptance of the PHOBOS apparatus to explore the correlation between energetic charged particles and all the other particles in the event, revealing more details about the nature of both the partonic energy loss and the dense medium.

\section{The PHOBOS detector system}

The PHOBOS apparatus consists of highly segmented silicon sensors, various scintillator and Cherenkov detector arrays and calorimeters. Figure 2.1 shows the schematic drawing of the complete layout.

The collision point is surrounded by the two arms of the multi-layered silicon Spectrometer, that are situated in a double dipole magnetic field. They have a pseudorapidity acceptance for charge particle tracking and momentum determination of $0<\eta<1.5$, although their azimuthal angle coverage is limited. The multiplicity array is a single layer of silicon detectors, and has two structural parts: the tube-like Octagon detector covers $|\eta|<3.2$ and the full azimuthal angle range. The six silicon Ring detectors placed perpendicularly to the beam-line extend the longitudinal coverage up to $|\eta|=5.4$.
The scintillator Paddle detectors are two segmented disks on both sides of the interaction point, covering the $3<|\eta|<$ 4.5 range. They are used to trigger on collisions, and to sort the triggered heavy ion events into centrality classes based on the total amount of energy deposited in the scintillators.

Particle identification is based on three techniques depending on the momentum range. Particles up $p_{T}=200$ $\mathrm{MeV} / c$ can be identified by their complete absorption in the first detector layers. The specific energy loss measured in the silicon sensors can be utilized to separate particle species up to $p_{T}=1.4 \mathrm{GeV} / c$. Particles with higher transverse momentum are identified via their simultaneous determination of momentum and velocity, measured by the scintillator Time of Flight walls.

Further details of the detector system can be found in [1].

\section{Charged hadron yields and their scaling properties}

One of the most important discoveries of the Relativistic Heavy Ion Collider (RHIC) is that high-energy partons interact strongly and lose a significant fraction of their energy as they traverse the high density medium produced in nuclear collisions. The energy loss can be inferred from the measurement of charged hadron transverse momentum spectra. Figure 3.1 shows examples of charged hadron spectra measured with the PHOBOS Spectrometer at $\sqrt{s_{N N}}=200 \mathrm{GeV}$ collision energy, in $\mathrm{Au}+\mathrm{Au}$ [2] and $d+\mathrm{Au}$ [3] collisions. In both cases, the spectra are measured in various classes of collision centrality, which are labelled with the relative percentage interval of the total inelastic cross section $(0 \%$ refers

Fig. 2.1 The schematic view of the PHOBOS detector system

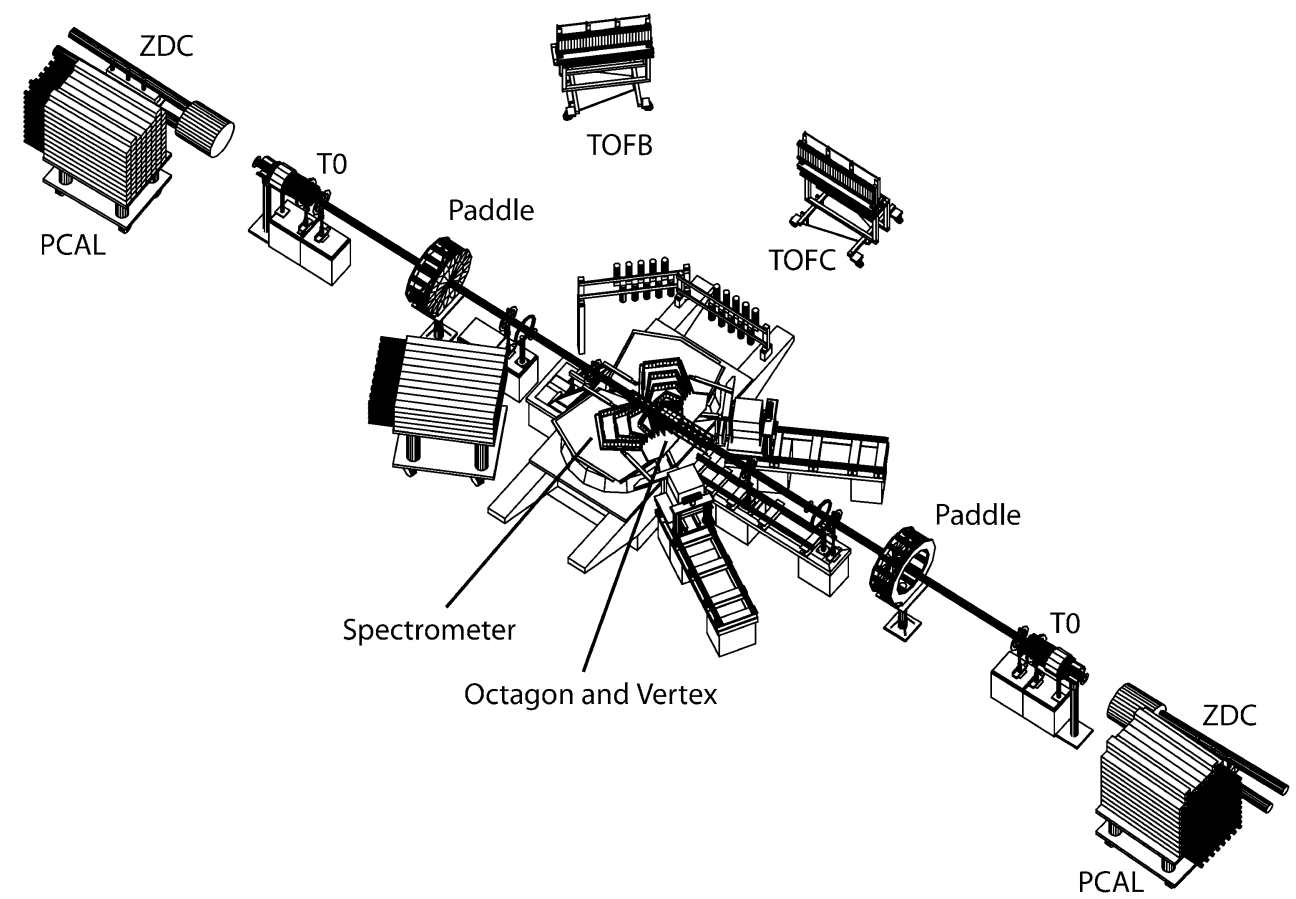


Fig. 3.1 Inclusive charged particle transverse momentum spectra measured in $\mathrm{Au}+\mathrm{Au}$ (left panel) and $d+\mathrm{Au}($ right panel) collisions at 200 $\mathrm{GeV} /$ nucleon center-of-mass energy
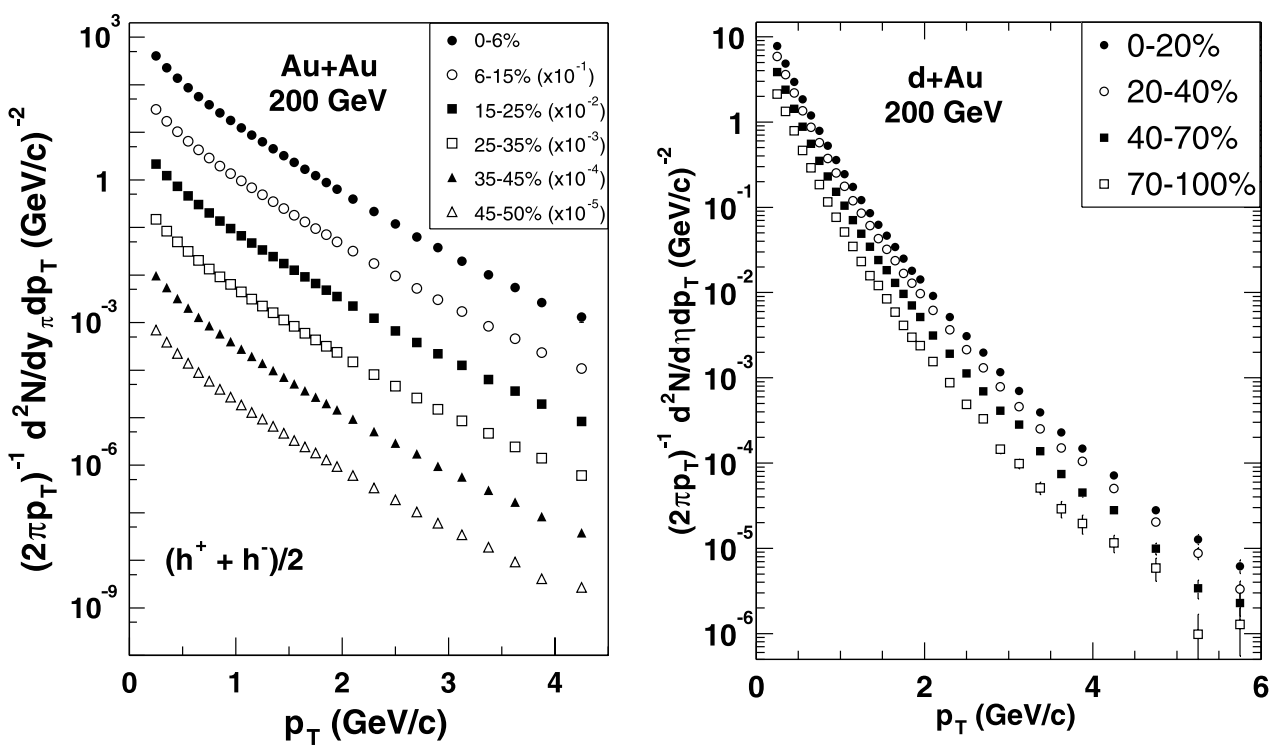

to the most central collision). In case of the $d+\mathrm{Au}$ data, a special high- $p_{T}$ trigger was utilized to extend the statistical $p_{T}$ reach.

The significant difference between the shapes of the $p_{T^{-}}$ spectra in $\mathrm{Au}+\mathrm{Au}$ and $d+\mathrm{Au}$ collisions is already visible on Fig. 3.1, even using logarithmic vertical scales. A much more precise way to quantify these differences is to compare the ratios of spectra between proton-proton collisions (as a reference) and collisions of more complex systems. These ratios are called nuclear modification factors and are defined as:

$R_{A A}\left(p_{T}\right)=\frac{\sigma_{p p}^{\text {inel }}}{\left\langle N_{\text {coll }}\right\rangle} \frac{d^{2} N_{A A} / d p_{T} d \eta}{d^{2} \sigma_{p p} / d p_{T} d \eta}$,

where $\left\langle N_{\text {coll }}\right\rangle$ is the mean number of binary nucleon-nucleon collisions in the Glauber model, characterizing the given centrality class of the data. Without any nuclear effects $R_{A A}=1$ is expected at high $p_{T}$.

Figure 3.2 shows the nuclear modification factors, $R_{d \mathrm{Au}}$, for charged hadrons in four centrality classes of $d+\mathrm{Au}$ collisions at $\sqrt{s_{N N}}=200 \mathrm{GeV}$ as a function of transverse momentum [3]. The $R_{\mathrm{AuAu}}$ nuclear modification factor for $\mathrm{Au}+\mathrm{Au}$ events at the same energy is also plotted for comparison with the solid line on the bottom right panel (corresponding to the most central collisions). While there is a strong suppression (by a factor of 4-5) of the hadron yields at high $p_{T}$ in the heavy ion data, the $R_{d \text { Au }}$ factors are around (or slightly exceed) unity for $p_{T}>3 \mathrm{GeV} / c$. Since the partons scattered at high- $p_{T}$ only have to traverse cold nuclear matter to hadronize in the $d+$ Au case, the result is an evidence that the observed suppression is caused by a final state effect in heavy ion collisions, which is connected to the presence of the created high density medium. Due to

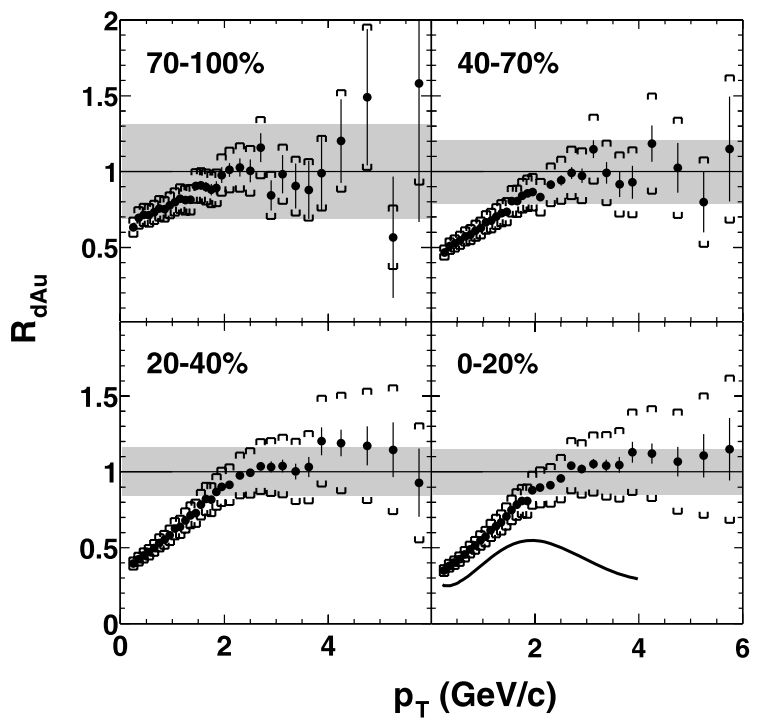

Fig. 3.2 Nuclear modification factors, $R_{d \mathrm{Au}}$, as a function of transverse momentum for charged hadrons in four centrality classes of $d+$ Au collisions at $\sqrt{s_{N N}}=200 \mathrm{GeV}$. The solid line on the bottom right panel (most central collisions) shows the $R_{\mathrm{AuAu}}$ nuclear modification factor for $\mathrm{Au}+\mathrm{Au}$ collisions at the same collision energy. Error bars stand for statistical, brackets for systematic errors, and the gray bands are scale errors common to all data points

the steeply falling transverse momentum spectrum, the parton energy loss at high $p_{T}$ manifests in the presented suppression, where particle yields in $\mathrm{Au}+\mathrm{Au}$ and $p+p$ collisions are compared at the same $p_{T}$. Similar results are also obtained by the PHENIX and STAR experiments at RHIC [4, 5].

Once the suppression of high- $p_{T}$ hadron yields is established in the heavy ion data, the more detailed na- 
Fig. 3.3 Nuclear modification factor, $R_{A A}$, in bins of $p_{T}$ versus $N_{\text {part }}$ at

$\sqrt{s_{N N}}=200 \mathrm{GeV}$, for $\mathrm{Cu}+\mathrm{Cu}$ (filled symbols) and $\mathrm{Au}+\mathrm{Au}$ (open symbols) [2,6]. The gray band in the first panel represents the relative uncertainty on $\left\langle N_{\text {coll }}\right\rangle$ and the solid lines show the effect of this uncertainty on $R_{A A}$. At high $p_{T}$, bands represent predictions of a parton quenching model [7]

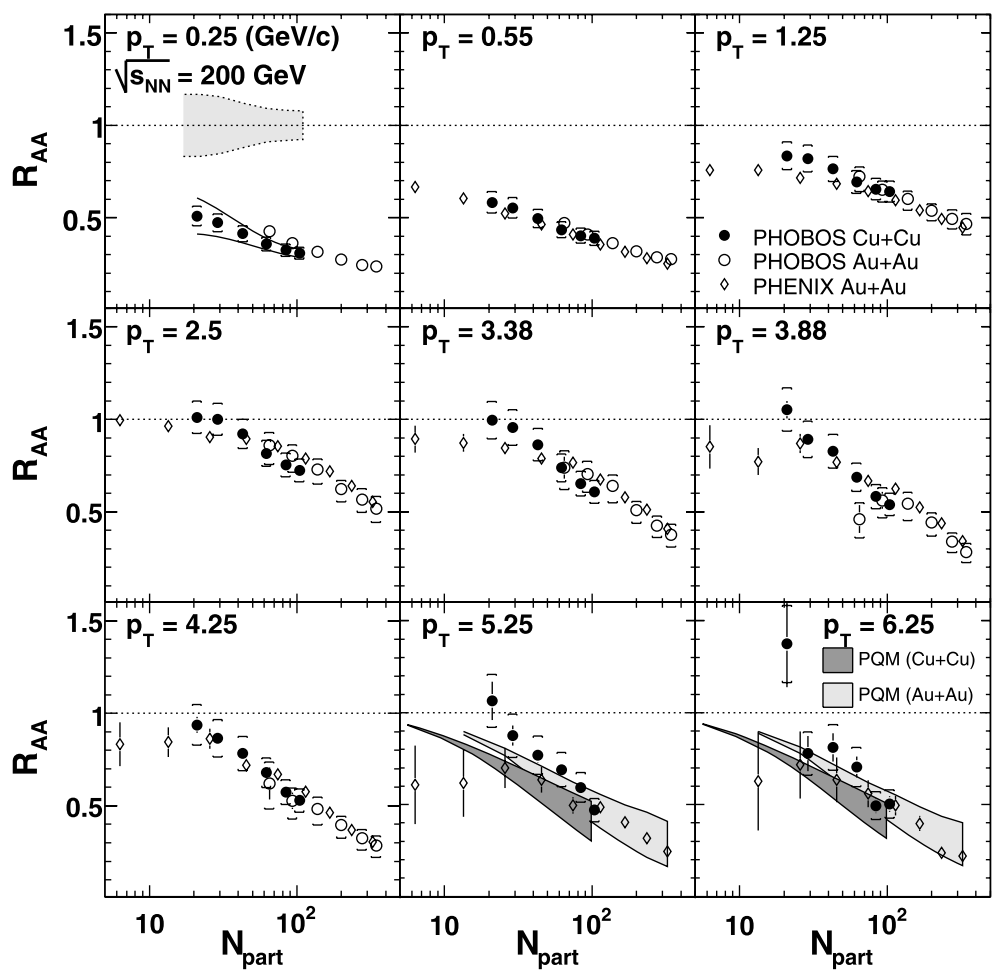

ture of the energy loss can be studied by comparing various centralities, beam energies and nuclear sizes. The PHOBOS collaboration has pointed out a number of remarkable scaling and factorization features unifying large sets of data while conducting these systematic comparisons.

An example of such scaling behavior can be seen on Fig. 3.3, where two different colliding systems, $\mathrm{Cu}+\mathrm{Cu}$ (closed symbols) and $\mathrm{Au}+\mathrm{Au}$ (open symbols) collisions are compared at $\sqrt{s_{N N}}=200 \mathrm{GeV}$ energy [8]. The nuclear modification factors, $R_{A A}$, are plotted as a function of the mean number of participants, $\left\langle N_{\text {part }}\right\rangle$, in the respective centrality bins. The various panels correspond to different transverse momentum values. Because of the large difference in the $\mathrm{Cu}$ and $\mathrm{Au}$ atomic mass, the same number of participant nucleons (same collision zone volume) implies very different collision geometries in the two cases: a thin, elongated collision zone in the $\mathrm{Au}+\mathrm{Au}$ collision, and a thicker, more spherical one in the $\mathrm{Cu}+\mathrm{Cu}$ collision. Therefore, a smaller suppression is expected in $\mathrm{Au}+\mathrm{Au}$ collisions, where the nuclear overlap zone is thin and elongated, corresponding to a smaller average path length to be traversed by the high- $p_{T}$ parton. On the contrary, the measured $R_{A A}$ values are consistent between the two systems, forming a universal $R_{A A}$ vs. $N_{\text {part }}$ curve that depends on $p_{T}$ but does not depend on the size of the colliding nuclei (i.e. the shape of the collision zone).
Another way to study the evolution of charged hadron yields with centrality is to compose the ratio of $p_{T}$ spectra in any given centrality bin and in the most central bin for heavy ion events, after normalizing them with their respective $\left\langle N_{\text {part }}\right\rangle$ values. The result quantifies the deviation of the shape of the spectrum from the most central one. It was shown that, surprisingly, the centrality evolution described this way is the same for $\mathrm{Au}+\mathrm{Au}$ collisions measured at $\sqrt{s_{N N}}=62.4 \mathrm{GeV}$ and $200 \mathrm{GeV}$ collision energy within errors [9], although variations in $R_{A A}$ as a function of energy, centrality and $p_{T}$ are much larger. This observation is an example of the factorization features observed in the inclusive charged hadron spectra.

Scaling behavior is not limited to results on non-identified particles. Figure 3.4 shows the transverse mass spectra of identified particles measured in $d+\mathrm{Au}$ collisions at $\sqrt{s_{N N}}=200 \mathrm{GeV}$ [10] and in $\mathrm{Au}+\mathrm{Au}$ collisions at $\sqrt{s_{N N}}=62.4 \mathrm{GeV}$ [11]. In this case, the $m_{T}$ spectra of various particle species have similar shapes in the more elementary $d+\mathrm{Au}$ collisions (although the absolute yield for the strange hadrons is suppressed), while the $m_{T}$ scaling is broken in heavy ion collisions. This observation shows another evidence-namely, the collective expansion-for the significant role the created medium plays in heavy ion collisions. The expansion of the medium introduces a massdependence to the shapes of the transverse mass spectra. 
Fig. 3.4 Invariant transverse mass, $m_{T}=\sqrt{m^{2}+p_{T}^{2}}$, spectra of identified particles in $d+\mathrm{Au}$ collisions at $\sqrt{s_{N N}}=200 \mathrm{GeV}$ (left panel, positive particles) [10] and in $\mathrm{Au}+\mathrm{Au}$ collisions at $\sqrt{s_{N N}}=62.4 \mathrm{GeV}$ (right panel) [11]
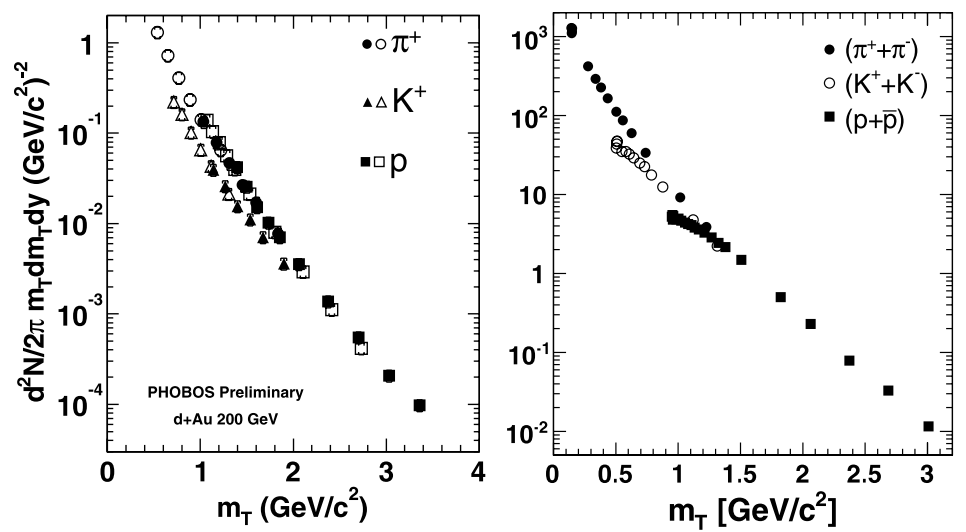

\section{Correlations with a high- $p_{T}$ particle}

The effect of the large amount of energy lost by energetic partons traversing the medium is also observed in azimuthal correlations where back-to-back high $p_{T}$ particles disappear in central $\mathrm{Au}+\mathrm{Au}$ collisions [12]. In these measurements, a high- $p_{T}$ trigger particle is selected from a given heavy ion event, and some, or all of the other particles in the same event are called associated particles, as sketched in Fig. 4.1. The correlation between the direction of the trigger and associated particles is measured, usually both in the azimuthal angle $(\phi)$ and pseudorapidity $(\eta)$ direction.

For associated particles selected to have high momentum, the behavior is consistent with the surface emission of jets, caused by the presence of an opaque medium that completely absorbs those jets directed at the interior.

However, the energy and momentum of the away-side jet must be present in the final state due to the energymomentum conservation, and it is necessarily carried by the associated particles. Therefore, there is a strong motivation to study the correlations between high $p_{T}$ triggers and lower $p_{T}$ associated particles. At mid-rapidity, data collected at RHIC show several nontrivial features in the structure of those correlation functions. Not only a broadening of the now reëmergent away-side structure in $\Delta \phi$, relative to $p+p$

Fig. 4.1 The sketch of the two particle azimuthal angle correlation measurement. The thick arrow represents the high- $p_{T}$ trigger particle, while the other arrows depict associated particles, in a plane perpendicular to the beam. The ellipse symbolizes the azimuthal angle distribution (averaged over many events)

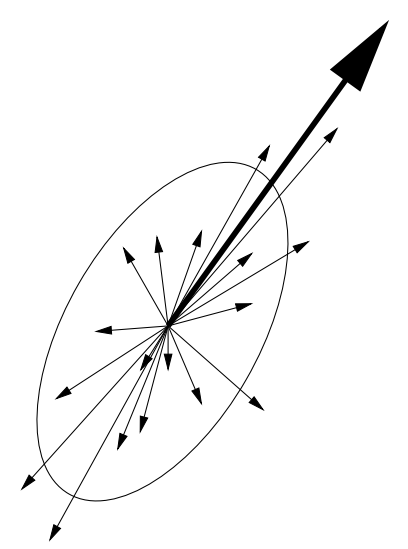

is observed [13, 14], but strikingly, the existence of an enhanced correlation near $\Delta \phi \approx 0$ is also found. This structure extends over several units of pseudorapidity, and has been called the 'ridge' [15]. Although the ridge at mid-rapidity has been qualitatively described by a diverse assortment of proposed mechanisms using various theoretical approaches [16-22], the precise origin of the structure is still not well understood. In this context, the goal of the PHOBOS collaboration is to use the uniquely broad pseudorapidity acceptance of the PHOBOS multiplicity detector array to measure the ridge structure (and its dependence on event centrality) at large relative pseudorapidity $(\Delta \eta)$, and therefore constrain the possible interpretations of particle production correlated with high $p_{T}$ trigger particles.

The silicon Spectrometer was used to select charged trigger tracks with $p_{T}>2.5 \mathrm{GeV} / c$ within the longitudinal acceptance of $0<\eta^{\text {trig }}<1.5$. Associated particles that are able to traverse the beryllium beam-pipe $\left(p_{T}>4 \mathrm{MeV} / c\right.$ at $\eta \approx 3, p_{T}>35 \mathrm{MeV} / c$ at $\eta \approx 0$ ) are detected in the singlelayered silicon Octagon detector, covering the $|\eta|<3$ range. There is no additional $p_{T}$ selection for associated particles. Due to the design of the Octagon, there are certain inactive windows in the detector, but those are filled using the first layers of the Vertex detector and the Spectrometer arms.

The conditional yield of charged particles per trigger is calculated by taking the raw per-trigger distribution of sameevent pairs, $s(\Delta \phi, \Delta \eta)$, and dividing by the raw distribution of mixed-event pairs (the 'background'), $b(\Delta \phi, \Delta \eta)$, to remove random coincidences and effects of the detector acceptance. This ratio is calculated in $1 \mathrm{~mm}$ wide bins of vertex position along the beam-line, $v_{z}$, and averaged over the range $-15<v_{z}<10 \mathrm{~cm}$ :

$$
\begin{aligned}
\frac{1}{N_{\text {trig }}} & \frac{d^{2} N_{c h}}{d \Delta \phi d \Delta \eta} \\
= & B(\Delta \eta) \cdot\left[\frac{s(\Delta \phi, \Delta \eta)}{b(\Delta \phi, \Delta \eta)}\right. \\
& \quad-a(\Delta \eta)[1+2 V(\Delta \eta) \cos (2 \Delta \phi)]]
\end{aligned}
$$


The last term in (4.1) is due to the collective effect in heavy ion collisions called elliptic flow: the azimuthal angle distribution of produced particles is not uniform, but has a cosine-like modulation (to first approximation) with a strength quantified by its second Fourier-coefficient, $v_{2}$. This distribution is symbolized by the ellipse in Fig. 4.1. The orientation of the ellipse is correlated with the impact parameter vector in the given nuclear collision.

Elliptic flow is completely erased in the mixing of tracks and hits (associated particles) from different events, thus the background will be uniformly distributed in $\Delta \phi$. However, both the trigger and the associated particles will be distributed according to the above non-uniform distribution, thus the same-event pairs will also show a remaining flow modulation, which is to be subtracted from the correlation measurement. The remaining second-order modulation is denoted by $V(\Delta \eta)$, and can be approximated by the product of $\left\langle v_{2}^{\text {trig }}\right\rangle$ and $\left\langle v_{2}^{a s s o c}\right\rangle$. The magnitude of the elliptic flow is calculated according to a parameterization based on published PHOBOS measurements of $v_{2}$ as a function of $N_{\text {part }}, p_{T}$, and $\eta$ in $\mathrm{Au}+\mathrm{Au}$ events at $200 \mathrm{GeV}$, assuming a factorized form [23].

The $v_{2}$ of the trigger tracks is corrected for occupancy effects in the Spectrometer, and the $v_{2}$ of the associated hits is corrected for secondaries. Both of these effects tend to suppress the magnitude of the observed $v_{2}$. Finally, the scale factor $a(\Delta \eta)$ in (4.1) is introduced to account for the small difference in multiplicity, caused by the trigger bias, between signal and mixed-event distributions for a given centrality class. It is calculated using the zero yield at minimum (ZYAM) method [24] and only differs from unity by a few percent in all cases considered. The ZYAM method ensures that the subtracted correlation function is nonnegative, and has its minimum value is zero. $B(\Delta \eta)$ is simply the corrected, published single-particle distribution $(d N / d \eta)$ [25] convoluted with the properly normalized $\eta$ distribution of trigger particles.

The dominant systematic error in the correlation analysis comes from the uncertainty in estimating the magnitude of the flow modulation, $\left\langle v_{2}^{\text {trig }}\right\rangle\left\langle v_{2}^{\text {assoc }}\right\rangle$. This uncertainty is typically on the order of $15-20 \%$, and in central collisions it reaches $50 \%$, but there the subtracted flow modulation is quite small compared to the jet correlation obtained after the subtraction.

To highlight the effects of the hot, dense medium on correlated particle production, the PHOBOS $\mathrm{Au}+\mathrm{Au}$ data is compared to $p+p$ events simulated with the PYTHIA event generator [26]. The $p+p$ correlation is shown in Fig. 4.2(a), and its prominent features are a jet-fragmentation peak centered about $\Delta \eta \approx \Delta \phi \approx 0$ and an away-side structure centered at $\Delta \phi \approx \pi$ that is similarly narrow in $\Delta \phi$ but extended in $\Delta \eta$. The elongated longitudinal nature of this away-side bump is expected, since the hard scattering

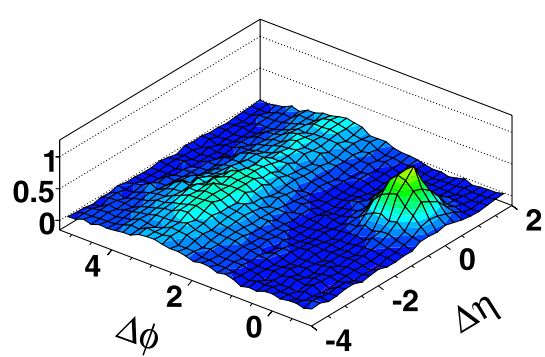

(a) $p+p$, PYTHIA

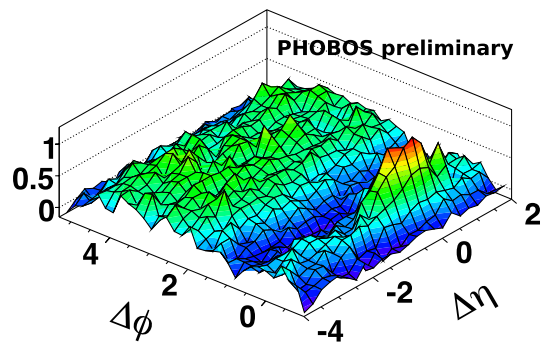

(b) $\mathrm{Au}+\mathrm{Au}$ central collisions $(0-30 \%)$

Fig. 4.2 Correlated yield per trigger particle using $p_{T}^{\text {trig }}>2.5 \mathrm{GeV} / c$, as a function of $\Delta \eta$ and $\Delta \phi$ for $200 \mathrm{GeV}$ (a) $p+p$ events generated by PYTHIA and (b) for $\mathrm{Au}+\mathrm{Au}$ collisions measured by PHOBOS

that has lead to the appearance of the high- $p_{T}$ trigger particle can involve partons with very different longitudinal momentum.

In central $\mathrm{Au}+\mathrm{Au}$ collisions, particle production correlated with a high $p_{T}$ trigger is strongly modified with respect to the $p+p$ events, as shown in Fig. 4.2(b). Not only is the away-side structure spectacularly broader in $\Delta \phi$, the near-side peak now sits on a pedestal, an unmistakable ridge of correlated partners extending continuously all the way to the edge of the detector acceptance at $\Delta \eta=4$. The observed elongation of the near-side ridge (in $\eta$ ) is much more challenging to interpret.

To examine the centrality dependence of the correlation structure, the correlated yield of associated particles as a function of $\Delta \phi$ was plotted in Fig. 4.3 in two $\Delta \eta$ slices: the top panels show the short range correlations defined as $|\Delta \eta|<1$, while the bottom panels show long range correlations in the $-4<\Delta \eta<-2$ interval. Data from central $\mathrm{Au}+\mathrm{Au}$ events $(0-10 \%)$ are plotted in the left column, and semi-peripheral data (40-50\%) can be seen in the right column. For comparison, the results from $p+p$ events generated by PYTHIA are also depicted with dashed lines. One can conclude that in semiperipheral $\mathrm{Au}+\mathrm{Au}$ events the near-side structure is compatible with the $p+p$ events, while the away-side broadening is still visible. However, for central $\mathrm{Au}+\mathrm{Au}$ events both structures exceed the $p+p$ events in magnitude, and there is a strong near-side peak even at the long $\Delta \eta$ range. 
Fig. 4.3 Per-trigger correlated yield for central $(0-10 \%$, left column) and semi-peripheral (40-50\%, right column)

$\mathrm{Au}+\mathrm{Au}$ collisions at $\sqrt{s_{N N}}=200 \mathrm{GeV}$ as a function of the $\Delta \phi$ distance between the trigger and associated particles. The upper row shows the short range $(|\Delta \eta|<1)$, the bottom row shows the long range $(-4<\Delta \eta<-2)$ parts of the correlation function. The dashed line represents the $p+p$ collisions from PYTHIA at the same collision energy
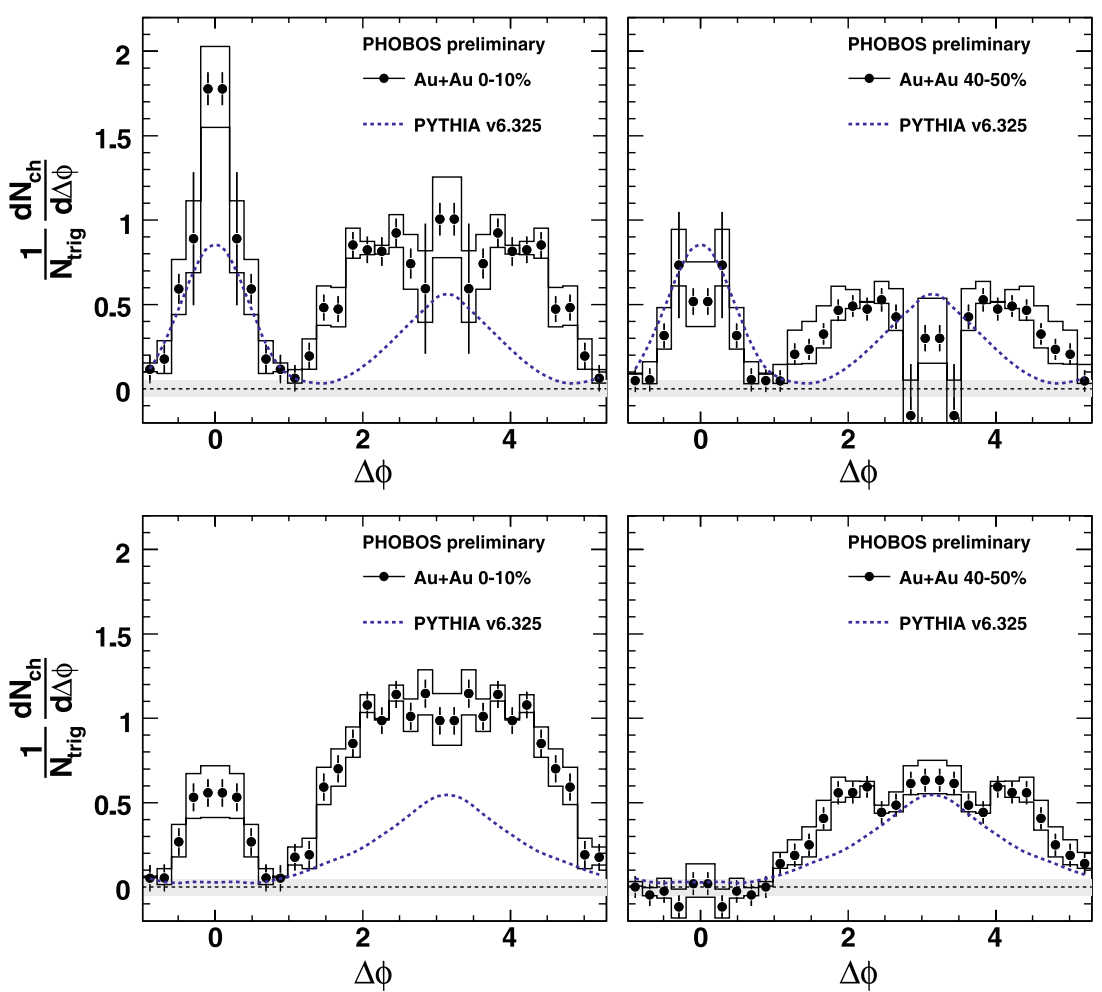

$\Delta \phi$
To examine the near-side structure more closely, the correlated yield is integrated over the $|\Delta \phi|<1$ region and plotted as a function of $\Delta \eta$ on the top panel of Fig. 4.4. for the $10 \%$ most central $\mathrm{Au}+\mathrm{Au}$ collisions. There is a significant and relatively $\Delta \eta$-independent correlated yield of about 0.3 particles per unit of pseudorapidity, even far away from the trigger. The prediction of the momentum kick model [27], which has been tuned to results of the STAR experiment with $\left|\eta^{a s s o c}\right|<1$ [14] and $2.7<\left|\eta^{a s s o c}\right|<3.9$ [28], is found to agree well with the experimental data. The momentum kick model postulates a parton rapidity distribution at the time of the jet-parton collision that is much broader than the rapidity distribution of final state hadrons. That would suggest that the extent of the ridge is sensitive to the earliest moments of the $\mathrm{Au}+\mathrm{Au}$ collision, and was proposed to be used as a probe of the initial parton rapidity distributions [29].

Finally, the centrality dependence of the average ridge yield far away from the trigger $(-4<\Delta \eta<-2)$ is shown on the bottom panel of Fig. 4.4. The ridge yield decreases towards more peripheral collisions, until it vanishes and becomes consistent with zero in the most peripheral bin analyzed (40-50\% centrality). While the present systematic errors do not exclude a smooth disappearance of the ridge yield as one approaches $p+p$ collisions, these preliminary data suggest the ridge may have already disappeared by the centrality class with $\left\langle N_{\text {part }}\right\rangle=80$.
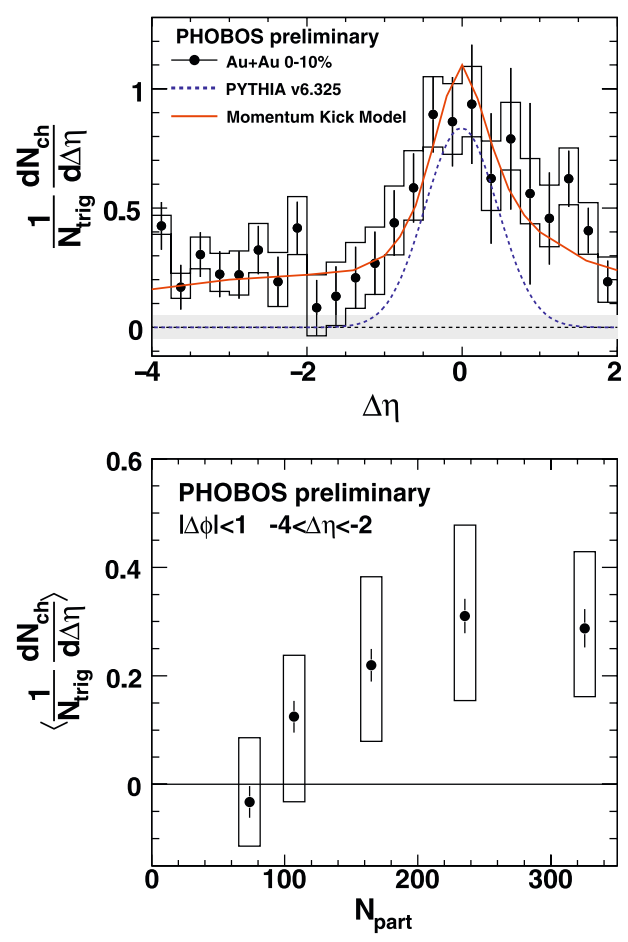

Fig. 4.4 Top panel: per-trigger correlated yield for central $\mathrm{Au}+\mathrm{Au}$ events $(0-10 \%)$ integrated over the near side $(|\Delta \phi|<1)$ compared to PYTHIA (dashed line) and to the momentum kick model prediction [27] (solid line) as a function of $\Delta \eta$. Boxes represent the systematic uncertainty. Bottom panel: average yield of the ridge as a function of $N_{\text {part }}$ in the $-4<\Delta \eta<-2$ range. Boxes represent the systematic errors 


\section{Summary}

An assortment of experimental results from the PHOBOS experiment at RHIC was presented on the hard probes of the medium created in ultra-relativistic heavy ion collisions. Charged hadron transverse momentum spectra are sensitive to the sizeable energy loss suffered by partons in this medium, and various ways to quantify this effect were described. Nuclear modification factors clearly show the quenching effect, and also obey certain scaling and factorization rules. These regularities unify various sets of data measured in nuclear collisions at different collision energy, system size and centrality.

Preliminary PHOBOS measurements of the ridge in the correlation structure between high- $p_{T}$ trigger particles and associated particles at small $\Delta \phi$ have been presented over a broad range of $\Delta \eta$. The fact that in central collisions the ridge extends to at least four units of rapidity away from the trigger is still a challenge to most theories that strive to quantitatively explain the nature of the jet-medium interaction. Further theoretical studies will be required to determine which proposed mechanisms are consistent with the broad extent of the ridge and its dependence on collision geometry.

Acknowledgements This work was partially supported by U.S. DOE grants DE-AC02-98CH10886, DE-FG02-93ER40802, DEFG02-94ER40818, DE-FG02-94ER40865, DE-FG02-99ER41099, and DE-AC02-06CH11357, by U.S. NSF grants 9603486, 0072204, and 0245011, by Polish MNiSW grant N N202 282234 (2008-2010), by NSC of Taiwan Contract NSC 89-2112-M-008-024, and by the Hungarian OTKA grant (F 049823) and the Zoltán Magyary Postdoctoral Fellowship.

\section{References}

1. B.B. Back et al. (PHOBOS), Nucl. Instrum. Methods A 499, 603 (2003)

2. B.B. Back et al. (PHOBOS), Phys. Lett. B 578, 297 (2004)

3. B.B. Back et al. (PHOBOS), Phys. Rev. Lett. 91, 072302 (2003)

4. S.S. Adler et al. (PHENIX), Phys. Rev. C 69, 034910 (2004)

5. J. Adams et al. (STAR), Phys. Rev. Lett. 91, 172302 (2003)

6. K. Adcox et al. (PHENIX), Phys. Lett. B 561, 82 (2003)

7. A. Dainese, C. Loizides, G. Paic, Eur. Phys. J. C 38, 461 (2005)

8. B. Alver et al. (PHOBOS), Phys. Rev. Lett. 96, 212301 (2006)

9. B.B. Back et al. (PHOBOS), Phys. Rev. Lett. 94, 082304 (2005)

10. G.I. Veres et al. (PHOBOS), J. Phys. G 30, S1143-S1147 (2004)

11. B.B. Back et al. (PHOBOS), Phys. Rev. C 75, 024910 (2007)

12. C. Adler et al. (STAR), Phys. Rev. Lett. 90, 082302 (2003)

13. A. Adare et al. (PHENIX), Phys. Rev. C 77, 011901(R) (2008)

14. J. Adams et al. (STAR), Phys. Rev. Lett. 95, 152301 (2005)

15. J. Putschke (STAR), J. Phys. G: Nucl. Part. Phys. 34, S679 (2007)

16. N. Armesto, C. Salgado, U. Wiedemann, Phys. Rev. Lett. 93, 242301 (2004)

17. C.B. Chiu, R.C. Hwa, Phys. Rev. C 72, 034903 (2005)

18. P. Romatschke, Phys. Rev. C 75, 014901 (2007)

19. A. Majumder, B. Müller, S.A. Bass, Phys. Rev. Lett. 99, 042301 (2007)

20. E.V. Shuryak, Phys. Rev. C 76, 047901 (2007)

21. V.S. Pantuev, arXiv:0710.1882 [hep-ph] (2007)

22. C.Y. Wong, arXiv:0712.3282 [hep-ph] (2007)

23. B.B. Back et al. (PHOBOS), Phys. Rev. C 72, 05190 (2005)

24. N.N. Ajitanand et al., Phys. Rev. C 72, 011902(R) (2005)

25. B.B. Back et al. (PHOBOS), Phys. Rev. Lett. 91, 052303 (2003)

26. T. Sjöstrand et al., Comput. Phys. Commun. 135, 238 (2001), using PYTHIA version 6.325 with default settings

27. C.Y. Wong, Private Communications

28. L. Molnar (STAR), J. Phys. G: Nucl. Part. Phys. 34, S593 (2007)

29. C.Y. Wong, Phys. Rev. C 76, 054908 (2007) 